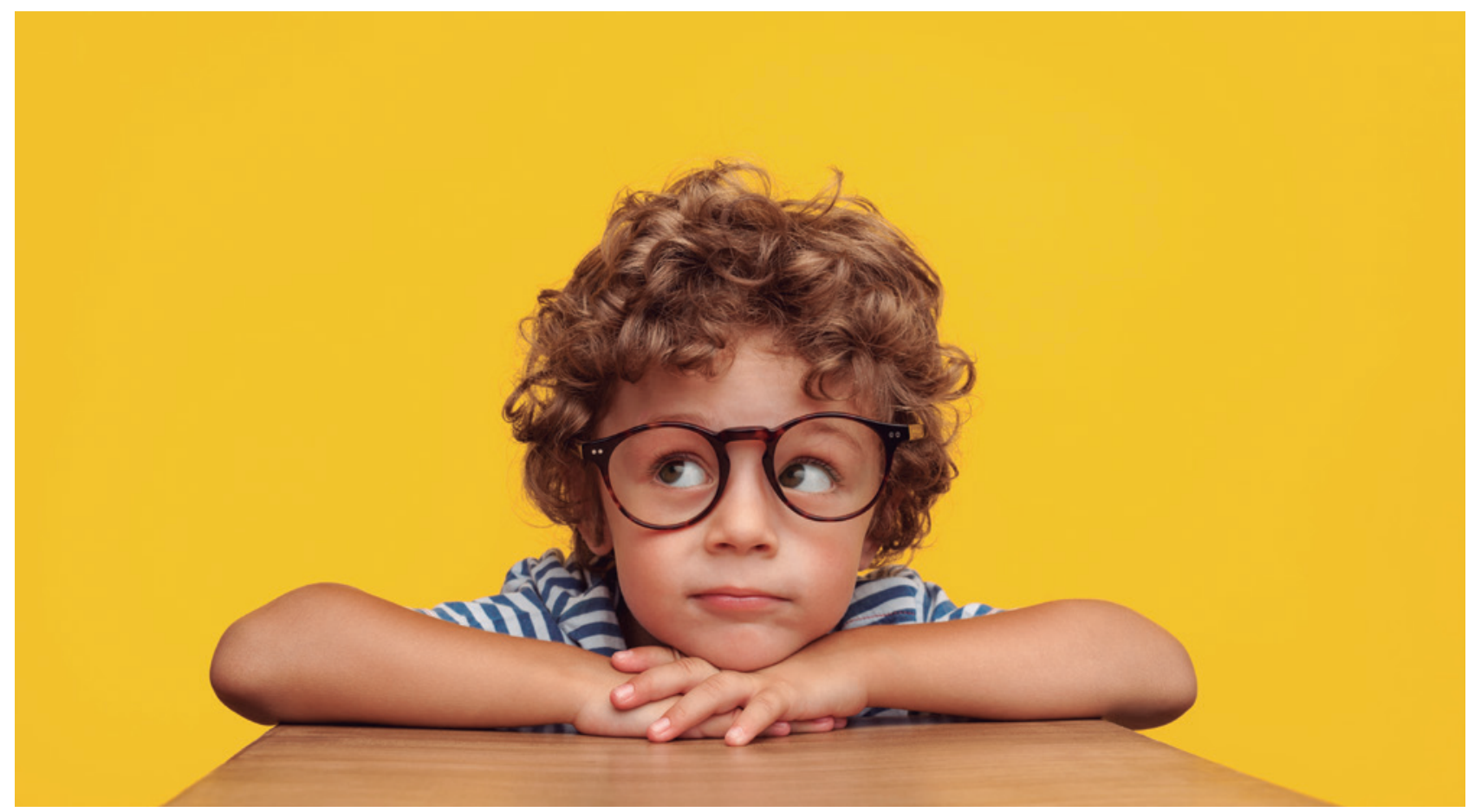

La educación

\title{
debe reimaginarse
}

Ante la profunda percepción de crisis en la mayor parte de los sistemas educativos de todos los países, son centenares las escuelas, redes de escuelas, instituciones y proyectos que exploran y hacen realidad metodologías innovadoras y nuevas escuelas. Hoy aparece un renovado interés por la educación como motor y herramienta básica para mejorar la humanidad. La educación debe reimaginarse, se debe transformar de abajo arriba, con la participación de toda la comunidad educativa. 
Hoy la sociedad está viviendo una verdadera primavera pedagógica vinculada a la innovación y al cambio en las escuelas. Efectivamente, ante la profunda percepción de crisis que experimentan la mayor parte de los sistemas educativos de todos los países, son centenares las escuelas, redes de escuelas, instituciones y proyectos en los que también a menudo están involucrados los gobiernos, que exploran y hacen realidad metodologías innovadoras y nuevas escuelas en edificios y tradiciones con frecuencia centenarios.

De esta forma, aparece un renovado interés por la educación como motor y herramienta básica para mejorar la humanidad. Y está ya en la agenda mundial todo lo que tiene que ver con la innovación y transformación del proceso de enseñanza y aprendizaje. Hoy se habla más que nunca de educación y de su sentido.

Por mi experiencia en el diseño y la puesta en marcha durante ocho años del proyecto Horizonte 2020 de los jesuitas de Cataluña, y por mi responsabilidad actual como director del equipo de www.riedulab.net, en estos últimos cuatro años he viajado a más de diecisiete países, de Europa y América, donde he entrado en contacto con centenares de equipos directivos y profesores de escuelas y universidades que están reaccionando frente a la crisis de la educación y están avanzando en la innovación educativa.

Por lo que atañe a este enriquecedor contacto y a los intercambios de experiencias y conocimientos realizados, he podido constatar un conjunto de nueve tendencias internacionales en aquellas escuelas y experiencias de muy distintos países que transitan por la transformación y el cambio educativo. Estas tendencias no son fruto tan solo de la investigación y el debate. Son fundamentalmente producto del intercambio de experiencias y de la observación de la realidad, de los sueños de equipos que no esperan leyes o señales para cambiar y que consideran que ningún obstáculo es lo bastante grande como para bloquearles.

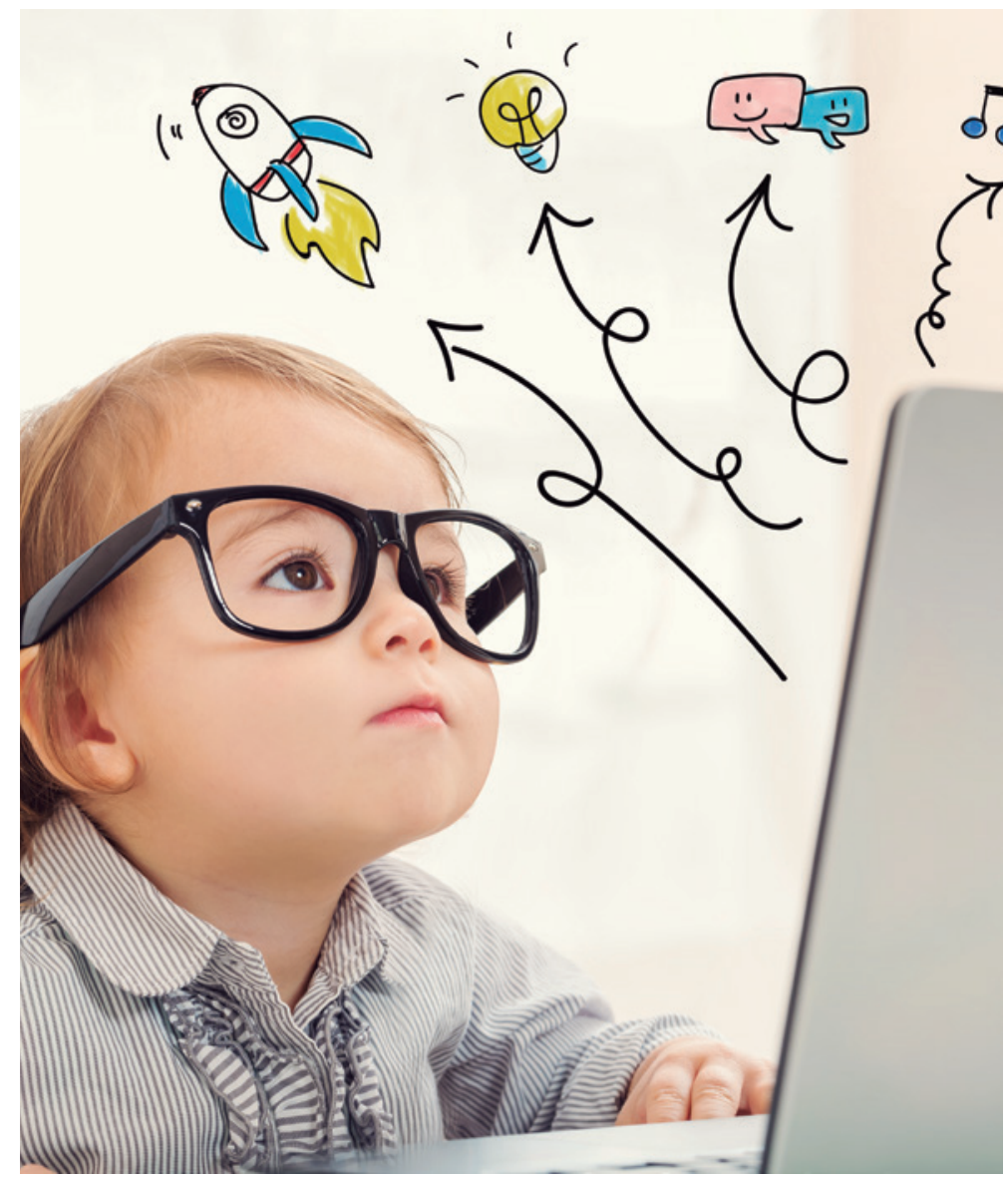

\section{Nueve claves para la transformación educativa}

Vamos a repasar, una por una, las nueve tendencias internacionales emergentes de la transformación educativa que he identificado y observado:

$\boldsymbol{\lambda}$ Un alumno que se ubica en el centro del proceso de enseñanza y aprendizaje es su protagonista y aprende haciendo. En el sistema tradicional que tratamos de transformar el centro lo ocupan el profesor, los contenidos y el currículo que se aspira a aprender. Todo ello debe descentrarse para conseguir poner en el centro al alumno, su desarrollo, sus intereses. Para que sea él quien de forma activa, mediante su trabajo individual y en equipo, vaya aprendiendo y desarrollándose mediante un planteamiento significativo y basado en la experiencia. Este camino nos ha de permitir plantearnos una mayor personalización, diversidad e inclusión.

入 Un profesor que cambia de rol y pasa de estar centrado en su materia o contenido a estar centrado en el desarrollo humano y personal del alumno. 


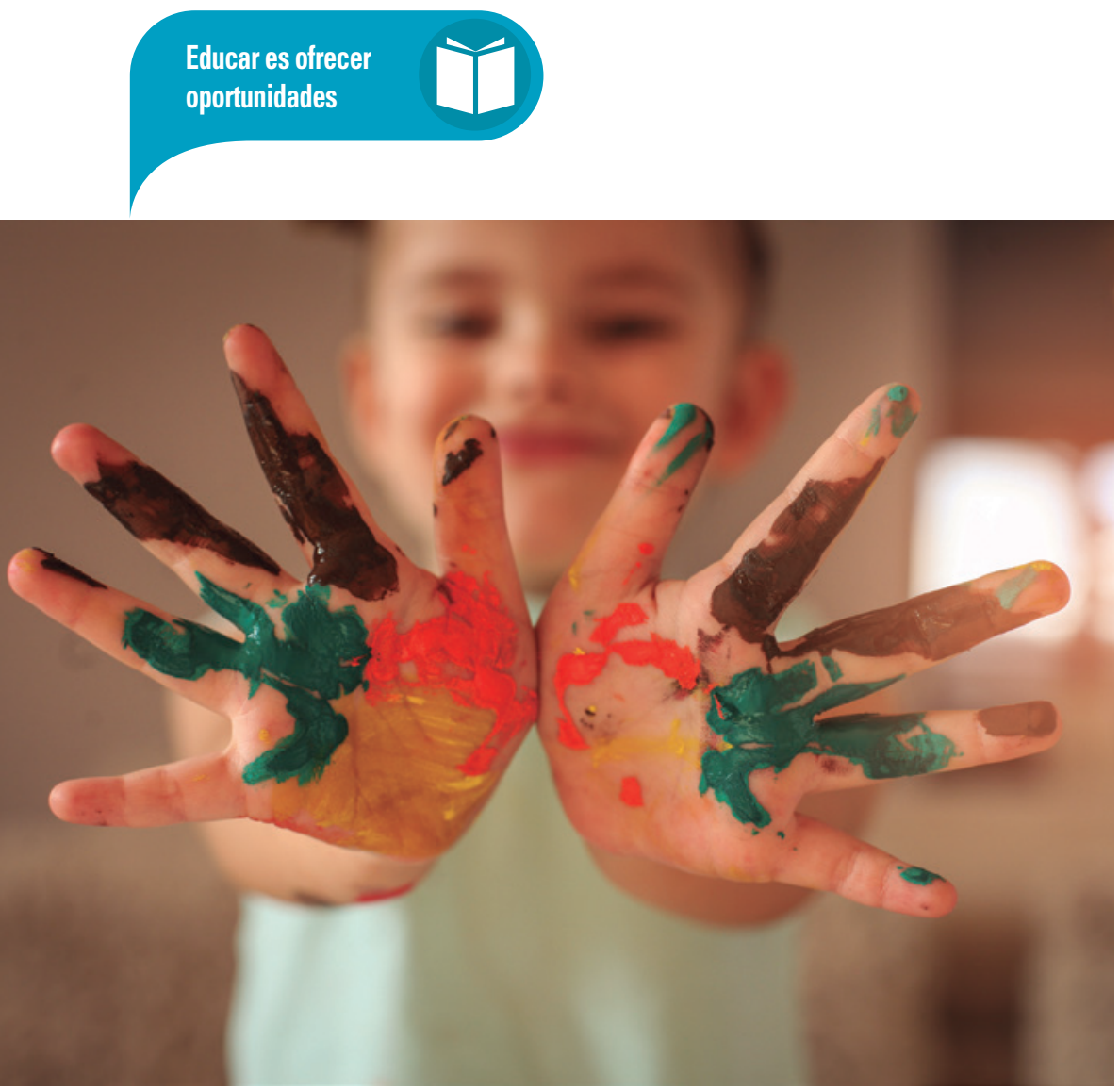

Un profesor que deja de estar en el centro del proceso de enseñanza y aprendizaje y centrado en su materia o foco curricular de forma individual y muy solitaria, y que transforma su rol de presentador y transmisor oral del conocimiento para devenir en un profesional centrado en el desarrollo y el crecimiento de la persona mediante una acción en equipo e interdisciplinar con otros profesores. Así, mediante el diseño de proyectos y entornos de trabajo y la dinamización de actividades en el aula, pero siempre cerca de los alumnos impulsa, interpela, guía y orienta sus trabajos individuales y en equipo. El profesor se convierte, pues, en un referente vital para sus alumnos.

$\boldsymbol{\lambda}$ Un aprendizaje basado en la comunidad educativa, en la relación personal, en la creación de vínculos y en el trabajo cooperativo.

El aprendizaje con los otros y en equipo, es fundamental para conocernos y construirnos como personas. Por tanto, el sistema de trabajo colaborativo debe ocupar una proporción significativa del tiempo lectivo de los alumnos mediante el trabajo por proyectos, la resolución de retos o problemas complejos, de forma que se establezca como referencia básica del proceso de enseñanza y aprendizaje la comunidad educativa. En ella, las agrupaciones de alumnos y profesores pueden variar combinando grupos grandes, grupos naturales o grupos pequeños, pero primando, en todo caso, el aprendizaje entre iguales.

入 Un aprendizaje interdisciplinar basado en el planteamiento y la resolución de retos que superan las materias curriculares y están conectados con la vida y el contexto significativo que rodea al alumno.

El trabajo interdisciplinar para la resolución de problemas y retos vinculados a la vida real y al contexto de los alumnos, que utiliza además dinámicas participativas y lúdicas, permite una educación más integral y un aprendizaje más activo y dinámico. En este sentido, las evidencias científicas existentes vinculan el trabajo en equipo y la interdisciplinariedad a la creatividad, a la capacidad de iniciativa, a aprender y al trabajo con los demás. Todos ellos, aspectos básicos de la persona que queremos educar, y que son básicos para el futuro del alumno y su proyecto vital.

入 Una educación con mirada y sentido integrales dirigida a todas las inteligencias del alumno y orientada a impactar en el conjunto de la persona para ayudarla a conocerse y a construir su proyecto vital.

La educación y el proceso de aprendizaje se enfocan de forma integral, con una nueva mirada al conjunto de la persona para integrar todas las inteligencias del alumno y así desarrollar aptitudes, competencias, conocimientos y valores pertinentes con el modelo de persona a educar. En este sentido, el eje fundamental de la nueva mirada y el sentido integral de la educación es la búsqueda del desarrollo del proyecto vital del alumno para que pueda disponer de las herramientas fundamentales para devenir en un ciudadano activo en su entorno.

入 Una evaluación de los alumnos y de su progreso totalmente transformada y al servicio del objetivo de educar. 
La evaluación de los alumnos es un elemento fundamental que cambia completamente su dinámica y enfoque. Así la evaluación debe enfocarse de forma holística, cualitativa, informal y continua, y ha de estar al servicio del desarrollo de la persona y del aprendizaje. De esta forma, pasa a ser diagnóstica, formativa y sumativa, e incluye autoevaluación, coevaluación y heteroevaluación para tener un feedback constante y avanzar en los procesos metacognitivos. En consecuencia, más allá del enfoque y de su función, ha de cambiar también el boletín de notas para el alumno y las familias a fin de que explique y comunique el nuevo modelo educativo y la nueva forma de evaluar.

7 Un acompañamiento activo de los maestros y profesores en su cambio de rol.

Los maestros y profesores deben tener un acompañamiento activo para cambiar de rol, en un proceso que vaya más allá de la formación clásica conceptual y que asegure también el "aprender haciendo" y el compartir con otros docentes sus preocupaciones y avances. Sin una dedicación específica y temporal para que el profesor pueda reflexionar sobre su acción docente y transitar individual y colectivamente por el camino del cambio de metodología y de rol, es imposible realizar y asegurar un cambio educativo en condiciones. Esta es, sin duda, una inversión necesaria.

入 Una evaluación efectiva de las iniciativas innovadoras y de cambio basada en el análisis, la observación y la cuantificación del impacto realizado en el alumno.

Se hace imprescindible evaluar el impacto sobre los alumnos y sobre el modelo de persona a educar de todas las innovaciones educativas que se vayan decidiendo e implementando. Esta evaluación se debe plantear en el mismo momento del diseño del cambio que se quiere realizar, en un avance decidido hacia una pedago-

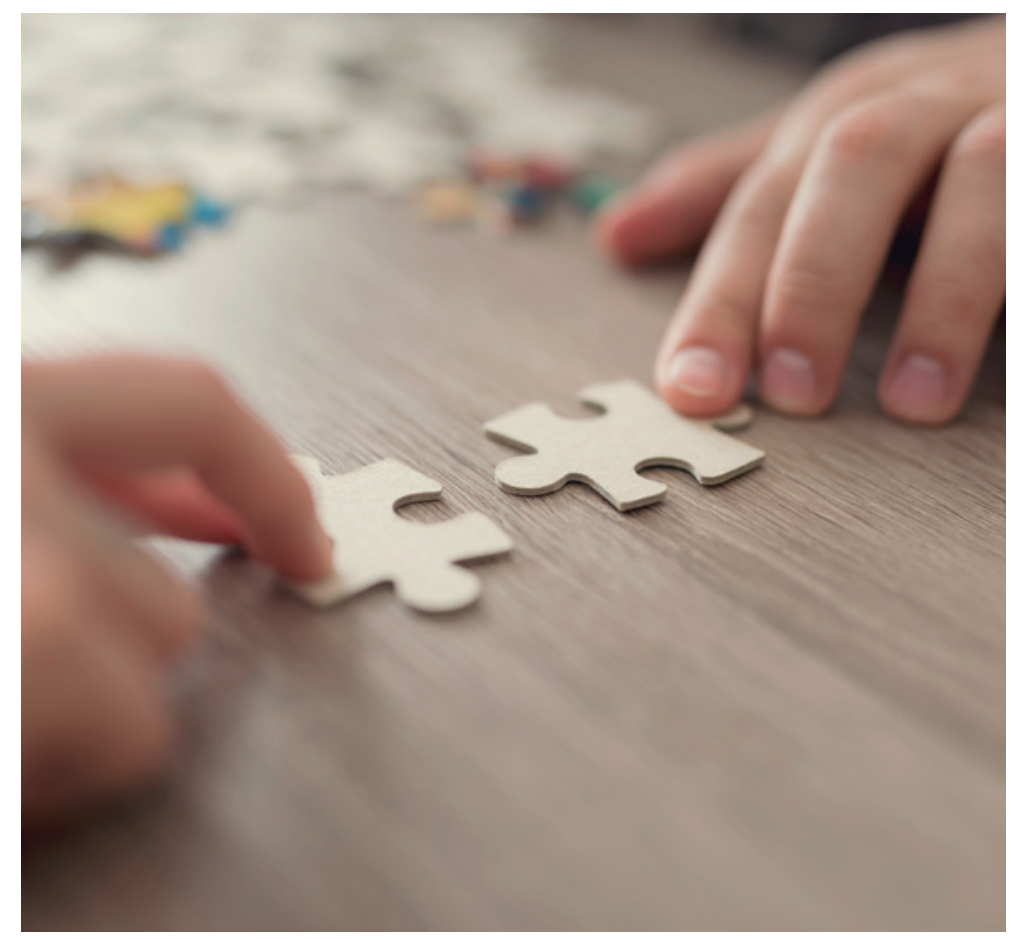

\section{Una educación con mirada y sentido integrales \\ dirigida a todas las inteligencias del alumno \\ y orientada a impactar en el conjunto de la \\ persona para ayudarla a conocerse y a construir \\ su proyecto vital}

gía más fundamentada en datos y en evaluaciones científicas, y en la construcción de una teoría para el cambio que posteriormente permita contrastarla y validarla. En este sentido, se va abriendo camino progresivamente la distinción entre resultado e impacto en el devenir del alumno dentro de la escuela.

入 Un trabajo abierto y en red con otras escuelas e instituciones para construir, de forma cooperativa, el camino de cambio.

Transformar una sola escuela aislada, por grande que sea, es muy difícil. Los diferentes contextos, el trabajo compartido y en red, la observación de las buenas prácticas de otros y compartir errores y aciertos, y hacerlo en red, es la mejor forma de aprendizaje colectivo que conocemos. Así, el trabajo abierto, experimental y en red se 


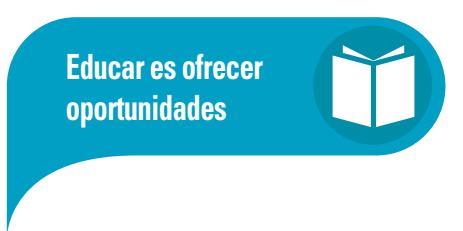

convierte en un gran instrumento para avanzar.

En todas las iniciativas de cambio e innovación que se llevan a cabo en estos momentos en el ámbito escolar, podemos observar la mayoría de las nueve tendencias que he descrito brevemente. Aunque no las llamen por este nombre o las bauticen con algún nombre técnico o específico.

Y ciertamente, aunque no hay un solo modelo por replicar, porque todos los contextos son distintos, como distintas son también las maneras de entender la educación por parte de cada institución y escuela, sí podemos reconocer estas nueve tendencias, de una forma u otra, en prácticamente todas las iniciativas innovadoras, que, generalizando, podemos denominar "pedagogías activas y globalizadoras".

\section{Dificultades para el cambio}

Sin embargo, también he podido constatar un conjunto dificultades y limitaciones que dificultan el cambio: ¿por dónde empezar?, ¿cómo involucrar a profesores, directivos, alumnos y familias en esa transformación?, ¿basta con añadir algunas innovaciones en las aulas o hace falta un cambio más profundo? Además, los miedos, por supuesto, y las limitaciones que nos imponemos, sobre todo las inercias, entorpecen cualquier cambio educativo que queramos poner en marcha.

Estas son las cinco condiciones fundamentales para la consolidación del cambio que, a mi modo de ver, concurren junto con las nueve tendencias antes citadas, y que son muy importantes para que no se desarticule o frustre el proceso y quede en una primavera sin continuidad:

त El liderazgo práctico y activo del equipo directivo que está al frente de un proceso integral de cambio. Generar un cambio en educación es un proceso sostenido en el tiempo que requiere una estrategia clara y un liderazgo fuerte, globalizador y compartido para llevar adelante la experiencia.

入 Sueño y convicción. Es decir, sueño para visualizar el cambio que quere- mos realizar, que es mucho más que la suma de pequeñas innovaciones, y convicción de que es posible más allá de la dificultad y complejidad que conlleva.

入 Superación de los marcos mentales de los equipos directivos. Los marcos mentales de los directivos son normalmente el principal freno para el cambio. Aunque a veces nos escudamos en otros frenos, como las leyes y normas, la verdad es que de las principales experiencias de cambio se concluye que el fundamental obstáculo que hay que superar son las creencias preestablecidas, los encargos inerciales y los marcos de actuación que tienen fijados los directivos de una escuela.

入 Identificación de la trasformación educativa como un proceso que requiere tiempo y que afecta a todos los elementos de la escuela como institución. Es decir, si transformar la educación y la escuela es un proceso de cambio personal, colectivo, organizativo y cultural, no podremos improvisarlo ni seguramente empezar directamente por realizar cambios en el aula, aunque este sea el objetivo final. Deberemos utilizar una metodología de cambio que permita una estrategia integral.

入 Unas condiciones favorables y promotoras de la administración o administraciones educativas pertinentes. Aunque, si bien es cierto que en este siglo los cambios reales y efectivos se establecen de abajo arriba, no es menos cierto que una administración educativa que aporte más recursos, no ponga trabas a la innovación, sino que la facilite e impulse mediante la simplificación de procesos y controles, y busque el fomento de la misma, asegura la generalización y una velocidad de crucero más favorable.

Es muy importante entender que estamos hablando de un "proceso" de transformación educativa. Es decir, no hablamos solamente de innovar o realizar cambios en las aulas, claro que los reali- 


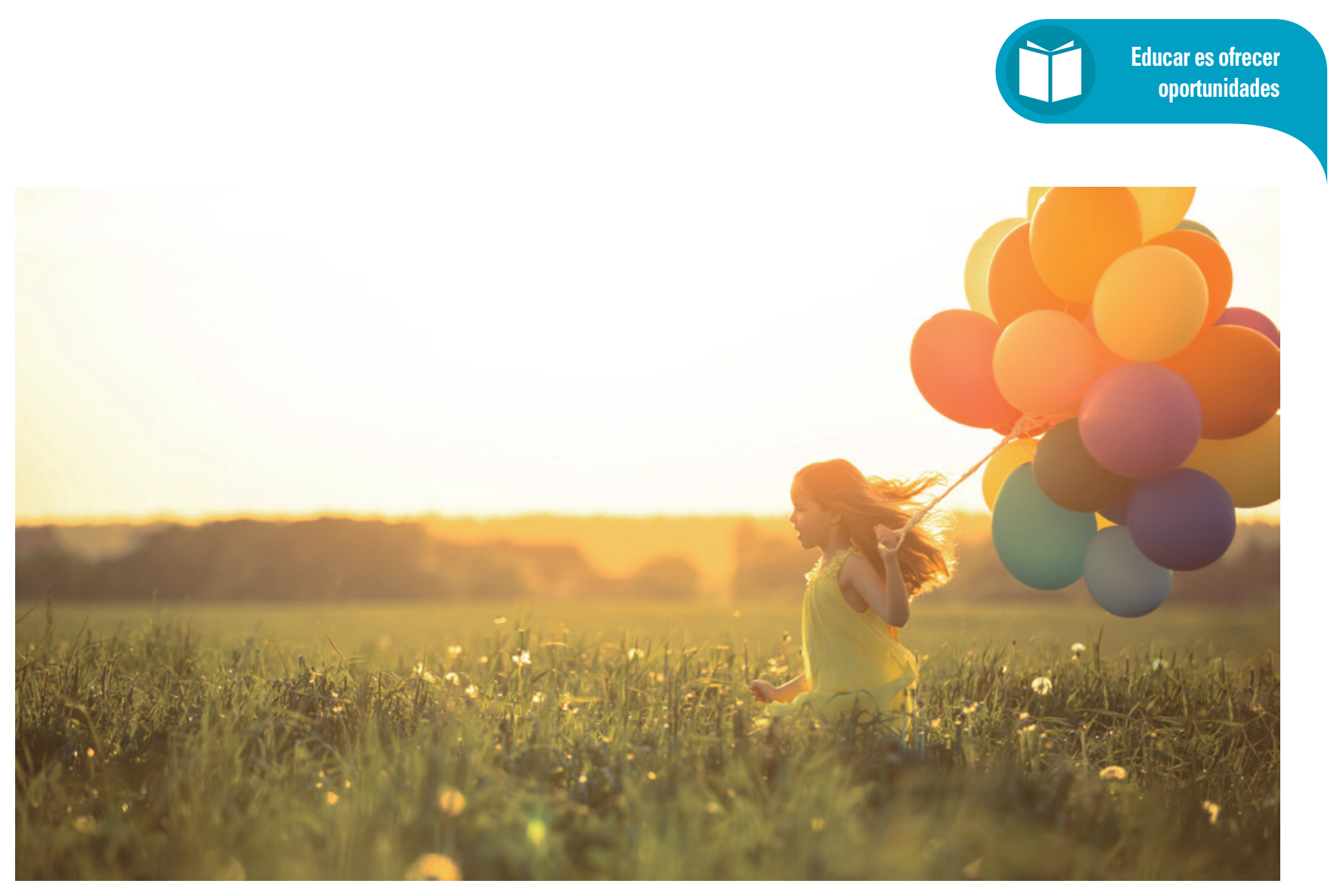

zaremos, pero nuestra intención, nuestro proyecto, es un cambio sistémico. Y, por tanto, un cambio de mirada, cultural, organizativo, de espacio físico. Es toda la escuela la que entra en un proceso de cambio profundo. Y para que esto ocurra, he observado que deben concurrir los cinco elementos citados.

\section{Pero ¿innovamos para adaptar o innovamos para transformar?}

Cuando hablamos de innovar, normalmente estamos planteando mejoras y retoques dentro del modelo clásico de enseñanza y aprendizaje y, por tanto, sin un planteamiento de cambio profundo. Es lo que se denomina "mejora continua". Las acciones de innovación son más bien operativas y con pocos riesgos estructurales o culturales. Sabemos el terreno que pisamos. Copiamos, adaptamos, rectificamos, reinterpretamos, sustituimos, apedazamos. Aunque al principio algunas innovaciones pueden apuntar alto, precisamente para no provocar un cambio más sistémico o porque chocamos con un techo de cristal que nos impide ir más allá, con frecuencia acaban adaptándose a las condiciones estructurales existentes. Como consecuencia de ello, las innovaciones que se acaban realizando, afectan un tanto por ciento marginal de la vida de aprendizaje del alumnado en el centro. Además, a menudo dependen, finalmente, de la buena voluntad del maestro o profesorado que debe aplicarlas en su grupo o aula.

Además, no requieren demasiado esfuerzo o energía colectiva, ni grandes decisiones o liderazgo. La formación es su instrumento fundamental, sin garantizar su aplicación una vez que esta formación se ha realizado. Normalmente se trata de una formación, además, que no interpela internamente, sino que mira hacia afuera. Tampoco se requieren grandes planes a medio o largo plazo. Más bien las innovaciones se plantean curso a curso y tienen mucho que ver con la propia evolución del sector educativo, en el que siempre hay algún movimiento de innovación basado en tecnologías o metodologías y productos. Ejemplos de esto en los últimos años han sido la incorporación de las TIC, los sistemas de calidad o mejoras metodológicas, como la introducción del trabajo por competencias.

Innovar es necesario e importante, pero quizá no siempre nos lleva a ese lugar futuro deseado de cambio y está muy conectado con el HACER.

\section{El proceso de cambio trasformador es sistémico}

Sin embargo, cuando hablamos de transformar, nos referimos a cambiar profundamente el proceso de enseñar y de aprender vigente en la escuela o en la 


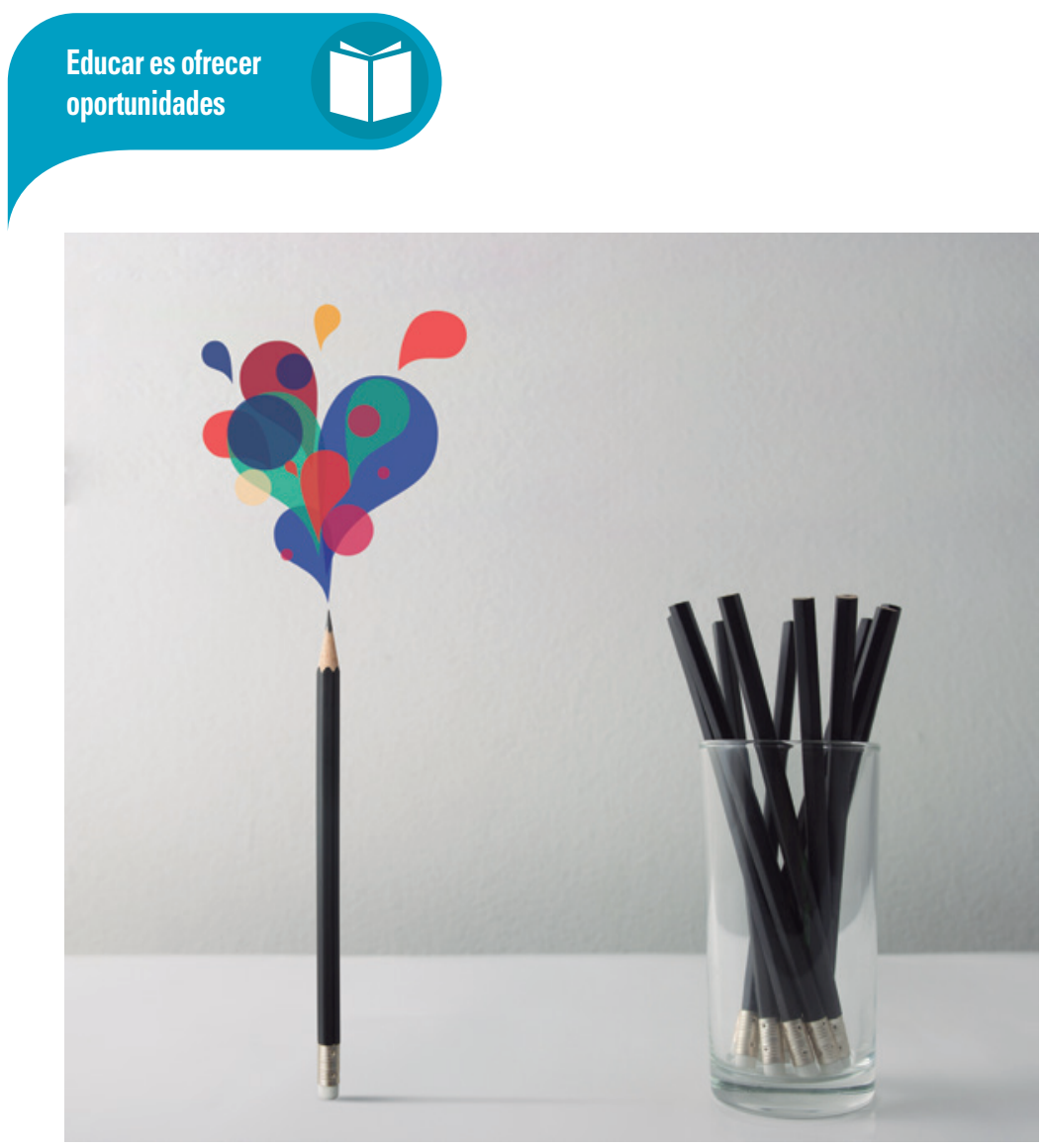

dosis de liderazgo, de convicción, de empoderamiento y de comunicación...

Pero, sobre todo, la transformación interpela a la persona, mira hacia dentro, conecta con la vocación para revitalizarla, invita a un cambio de mirada. Transformar conecta con el SER. En un proceso de transformación caben, en forma de prototipos y experiencias, muchas innovaciones específicas que difícilmente se pueden concretar o consolidar con el planteamiento de las acciones a corto plazo, porque normalmente el sistema las limita o las impide. Pero, en todo caso, su complejidad superior requiere una metodología que nos ayude a llevarla a cabo y que atienda todos los elementos que hay que tener en cuenta.

La acumulación de innovaciones puntuales más o menos ordenada o caótica no nos llevará jamás a una transformación profunda. Más bien, al hacerse de forma simultánea a las actividades que ya realizamos normalmente (que son muchas), nos puede llevar a un cierto estrés organizativo que, a medio plazo, puede facilitar la vuelta adonde estábamos antes de empezar.

La transformación nos puede dar una diferenciación muy importante de otros universidad para llegar a otro distinto: es decir, hablamos de un cambio sistémico. Se trata de rediseñar, de hacer prototipos, de concretar experiencias avanzadas de cambio, de ir más lejos. La transformación no es evolutiva o incremental. La transformación es disruptiva. Transformar tiene normalmente más riesgo a corto plazo, pero el hecho de requerir de una planificación a medio y largo plazo asegura mejor que lleguemos adonde queremos dentro de unos años. Para transformar, hay que definir una estrategia y un proceso, lo que significa poner más energía, tomar decisiones e identificar el talento interno y externo para alcanzar las metas planteadas. Implica, finalmente, un cambio cultural y organizativo, estructural, basado en una coalición y alianzas por el cambio que garanticen la realización de actuaciones que hacen salir de la zona de confort. Y, en conjunto, exige una fuerte proyectos educativos y una ventaja competitiva suplementaria. Por otra parte, hay que tener en cuenta que todo a nuestro alrededor se está transformando, y a una gran velocidad.

\section{Un modelo de transformación hacia una escuela inclusiva}

Si el lector ha llegado a este punto del artículo, lo que le agradezco profundamente, quizás ha tenido la duda de que la innovación y el cambio de los que hablo solo se los pueden permitir las escuelas que tienen recursos y que desarrollan su actividad en contextos favorecidos... En mi opinión, nada más lejos de la realidad.

Concibo la innovación educativa como un intento organizado y colectivo de transformar el proceso de aprendizaje de los alumnos (en una escuela o red de escuelas) para conseguir una educación integral (valores, competencias, aptitudes y 
contenidos curriculares) que les permitan desarrollarse como personas (proyecto vital) en el siglo en que les toca vivir. Realizar este proceso (que no consiste únicamente en introducir algún pequeño cambio) es muy complejo y requiere intervenir en todos los elementos del proceso educativo y con todos los actores de la comunidad educativa.

Y para impulsar este cambio es imprescindible una necesidad clara que apremie. Y en mi experiencia directa, basada en la transformación desarrollada, o indirecta, sustentada en las visitas a escuelas y organizaciones de distintos países, la necesidad de mejorar los resultados e impactos educativos en entornos socioeconómicos difíciles y complejos ha sido, es y será un motor de innovación transformadora que busca la inclusión social. Hay muchas experiencias en este sentido.

La investigación y la experiencia muestran que se pueden mejorar mucho los resultados educativos y, por tanto, avanzar decididamente hacia la inclusión social introduciendo innovaciones disruptivas en el proceso de enseñanza y aprendizaje. Porque los alumnos de menor nivel socioeconómico son los que más problemas tienen para adaptarse a un modelo de educación hoy caduco, que se diseñó en el siglo XIX para una minoría de nivel socioeconómico alto y que, sin cambios sustanciales, afortunadamente hemos extendido en los países occidentales a toda la población y a todos los niveles sociales.

Situar al alumno en el centro del proceso, asegurar que "aprende haciendo" mediante el trabajo colaborativo y que desarrolla proyectos interdisciplinares que integran conocimientos, valores, competencias y aptitudes, a la vez que incluye todos los elementos específicos personales y sociales que le permiten una vida plena para integrarse en la sociedad y transformar el mundo en que vive, es la mejor manera de llevar a cabo la inclusión social que queremos y deseamos. Y para ello, es imprescindible una innovación profunda que transforme la escuela y el aula.

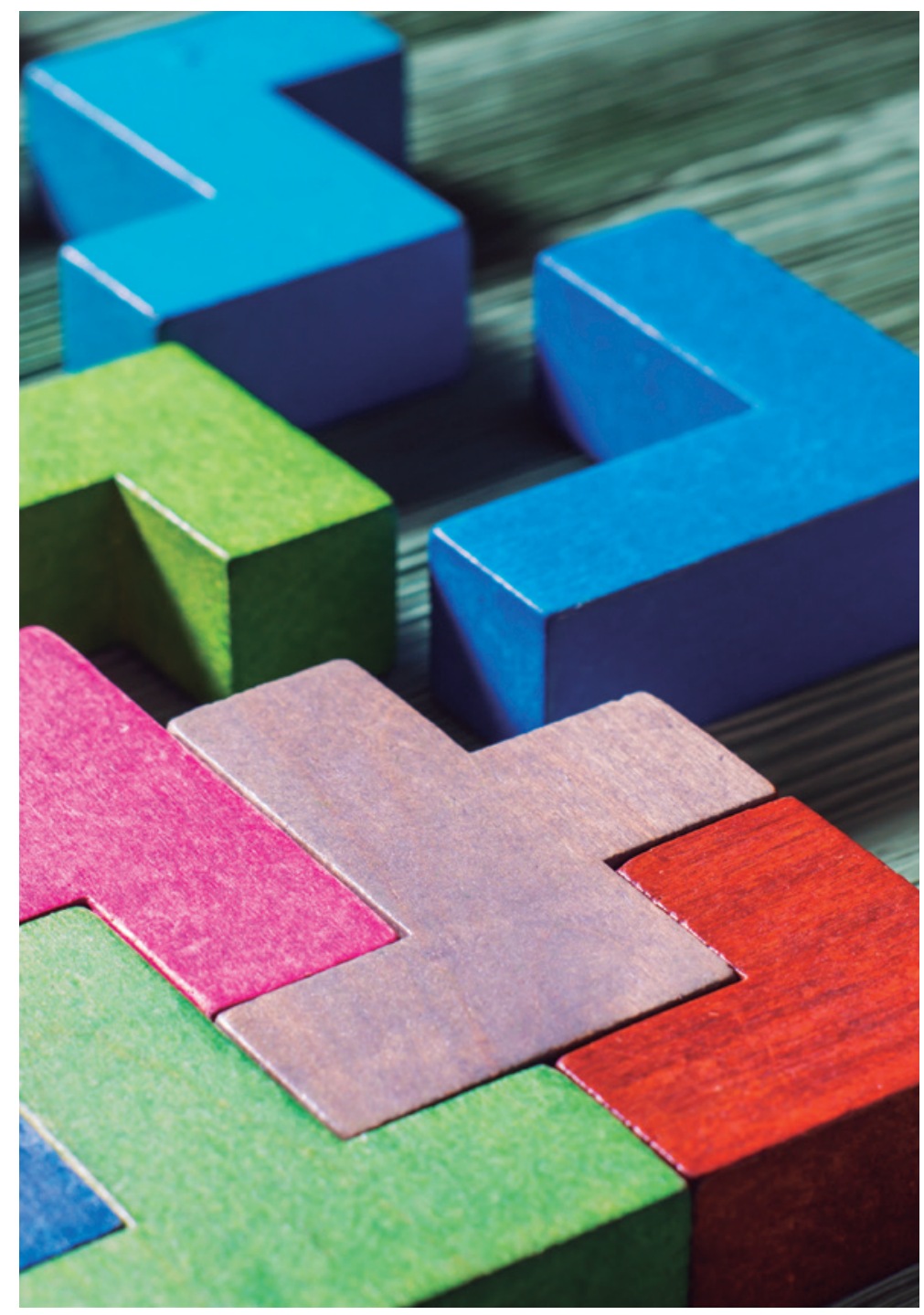

Asegurar una educación plena y la inclusión social implica innovar también en tres elementos clave del cambio educativo: el establecimiento de un vínculo con los alumnos, el trabajo directo con las familias y la integración de la educación formal con el tiempo libre. El vínculo con el profesor (más allá de la mera transmisión de contenidos) refuerza y empodera al alumno. Incorporar a las familias en el proceso de aprendizaje mediante nuevas estrategias asegura el impacto educativo. Tener una visión integrada y operativa del proceso de la educación formal y de la no formal es garantía de éxito en entornos socioeconómicos pobres.

Nos queda mucho camino por recorrer, pero inclusión social e innovación educativa son inseparables. Y personalmente no concibo la una sin la otra.

La educación debe reimaginarse. Y es una herramienta tan poderosa e impor- 


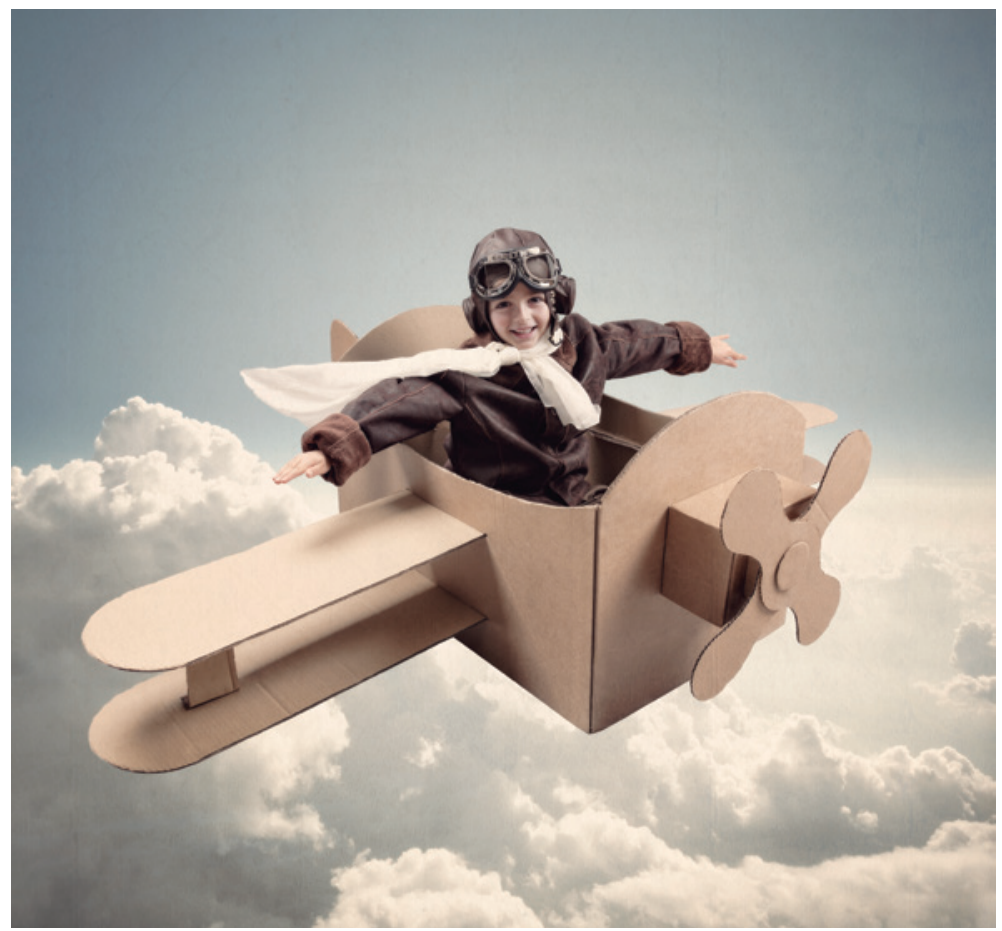

La educación debe reimaginarse. Y es

una herramienta lan poderosa e importante que no podemos dejarla en manos de la inercia o de la mera actividad por la actividad. La educación se debe transformar de abajo arriba con la participación de toda la comunidad educativa

tante que no podemos dejarla en manos de la inercia o de la mera actividad por la actividad. Tampoco podemos esperar que una nueva ley o norma la transforme. La educación se debe transformar de abajo arriba con la participación de toda la comunidad educativa.

A mi modo de ver, no debemos olvidar que la escuela, la universidad, la hacen las personas. Aunque a menudo hablamos de ellas como instituciones, en el fondo son personas que realizan muchas

\section{(D.) DARR SABER MÂS}

Aragay Tusell, X. (2017). Reimaginando la educación: 21 claves para transformar la escuela. Barcelona: Paidos. actividades con otras personas y para las personas que se están formando o educando. Y solamente la persona, cada una de ellas, puede decidir realizar un cambio educativo si lo hace libremente, si sueña y se compromete y se arriesga a dar el salto.

Y precisamente en el interior de las personas reside la principal fuerza y también la limitación más importante para realizar el cambio. La fuerza para la transformación se halla en la conexión entre la vocación y la mirada dirigida a la persona y al futuro. La limitación radica en los marcos mentales desajustados respecto al momento que vivimos y al futuro que adivinamos. $Y$, a menudo, estos marcos desajustados se expresan en forma de desconcierto, de conservadurismo, de perfeccionismo, de miedo o de hiperactivismo.

Con todo lo expuesto hasta aquí espero inspirarte para reimaginar la educación. Reimaginar tu escuela, tu aula. Espero ayudarte a actuar con conexión y sentido, y encaminándote hacia tu horizonte de cambio. Así, juntos, todos y todas los que soñamos transformar la educación, nos ayudamos y nos inspiramos.

Mi intención con este artículo es muy básica, me propongo no dejar indiferente. Provocar un impacto. Un cambio. Después, tú, que conoces tu propia realidad, podrás trazar tu propio camino del cambio y compartirlo con tu equipo y tu red. Porque solamente juntos podremos avanzar.

¡Ojalá avances por este camino! ¡Y ojalá lo podamos compartir! ¿Te apuntas? •

\section{HEMOS HABLADO DE}

\section{Innovación educativa; equidad; calidad educativa; liderazgo pedagógico; cambio social; escuela inclusiva.}

Este artículo fue solicitado por PADRES Y MAESTROS en diciembre de 2017, revisado y aceptado en marzo de 2018. 